\title{
Creación de una huerta medicinal para el fortalecimiento de las competencias lectoescrituras de los estudiantes de la Institución Educativa Técnica Departamental San José ${ }^{1}$
}

\section{Creation of a medicinal garden at the Departmental Educational Institution San José}

DOI: http://dx.doi.org/10.17981/cultedusoc.9.3.2018.08

\author{
Artículo de investigación. Fecha de recepción: 15/06/2018. Fecha de aceptación: 27/11/2018 \\ Juan Carlos Perlaza²; \\ Yudis Montagut, Jaquelina Madera, Kervis Sánchez, \\ Luz Gutiérrez, Idalid Torres, Celia Polo ${ }^{3}$ \\ IED San José sede Principal (Colombia) \\ jcpr@yahoo.es
}

Para citar este artículo:

Perlaza, J., Montagut, Y., Madera, J., Sánchez, K., Gutiérrez, L., Torres, I. y Polo C. (2018). Creación de una huerta medicinal para el fortalecimiento de las competencias lectoescrituras de los estudiantes de la Institución Educativa Técnica Departamental San José. Cultura. Educación y Sociedad 9(3), 73-78. DOI: http://dx.doi.org/10.17981/cultedusoc.9.3.2018.08

\section{Resumen}

La lectoescritura es una de las competencias bases de la formación de los estudiantes desde las primeras etapas de su vida, y la manera en la que se desarrolla depende de las metodologías que se implementan y la motivación e interés que se tenga por aprender a y desarrollarlas de manera adecuada, por lo tanto, el presente proyecto pretende fortalecer las competencias lectoescritoras de los estudiantes a través de la creación de la huerta medicinal, fomentando las actividades pedagógicas interdisciplinarias con la construcción de espacios naturales , específicamente plantas medicinales propias de la región. La investigación es de tipo cualitativo, de corte descriptivo, con un diseño de investigación acción. La población participante es de 200 estudiantes de la Institución Educativa Técnica Departamental San José, distribuidos en los cursos pertenecientes a la básica primaria y básica secundaria de la Institución, con edades que oscilaron entre los 4 y 17 años. Los instrumentos aplicados fueron la entrevista semiestructurada, observación participante la cual fue registrada a través de un diario de campo. Dentro de los resultados se puede inferir que se logró realizar el montaje del huerto medicinal en la Institución; además se educó a los estudiantes para que entendieran la importancia y función de las plantas medicinales, se potenciaron habilidades de escritura y literaturas asociadas directamente con la huerta medicinal.

Palabras clave: Huerta medicinal, competencias lector-escritoras, formación estudiantil.

\section{Abstract}

Literacy is one of the basic competences of the formation of students from the first stages of their life, and the way in which it develops depends on the methodologies that are implemented and the motivation and interest to be learned and to develop them. Therefore, this project aims to strengthen students' literacy skills through the creation of the medicinal garden, fostering interdisciplinary pedagogical activities with the construction of natural spaces, specifically medicinal plants native to the region. The research is qualitative, descriptive, with an action research design. The participating population is 200 students of the Departmental Educational Institution of San José, distributed in the courses belonging to the primary and basic secondary school of the Institution, with ages ranging from 4 to 17 years. Within the results it can be inferred that the assembly of the medicinal garden was achieved in the Institution; In addition, students were educated to understand the importance and function of medicinal plants. Writing skills and literature directly associated with the medicinal garden were promoted, however, interdisciplinary activities must continue to be carried out from the classroom to the interaction medium. With which students usually frequent.

Keywords: Medicinal garden, reader-writer skills, student training.

1 Este artículo ha sido derivado del Programa de Fortalecimiento de la Cultura Ciudadana y Democrática CT+I a través de la IEP apoyada en TIC en el Departamento de Magdalena: CICLON

2 Líder del Grupo de Investigación "Amigos de la investigación".

3 Docentes miembros del Grupo de Investigación "Amigos de la investigación", de la IED San José sede Principal.

- The author; licensee Universidad de la Costa - CUC

Cultura, Educación y Sociedad vol. 9 no. 3, pp. 73-78. Diciembre, 2018

Barranquilla. ISSN 2389-7724 Online 


\section{Introducción}

Como es bien sabido los medicamentos sintéticos poseen por lo general, demasiadas contraindicaciones y efectos secundarios que pueden afectar la salud de quien los ingiere, empeorando el estado de las personas que los consumen. Es por esto que la medicina natural representa una alternativa más saludable que las medicinas sintéticas, sin embargo, no se pretende con este proyecto promover que los habitantes de nuestra comunidad no ingieran las medicinas sugeridas por los médicos, pues ellos tienen un conocimiento sobre el malestar por el que está pasando el paciente y saben qué medicamento puede ayudarle a su mejoramiento. Se aprovecharon las plantas medicinales propias de nuestra región para llevar a cabo el huerto medicinal en la Institución Educativa Técnica Departamental San José de Sitio Nuevo, Magdalena.

\section{Huertas Medicinales}

Las plantas constituyen un recurso valioso en los sistemas de salud de los países en desarrollo. Aunque no existen datos precisos para evaluar la extensión del uso global de plantas medicinales, la Organización Mundial de la Salud (OMS) ha estimado que más del $80 \%$ de la población mundial utiliza, rutinariamente, la medicina tradicional para satisfacer sus necesidades de atención primaria de salud y que gran parte de los tratamientos tradicionales implica el uso de extractos de plantas o sus principios activos (Nogales, 2004). De acuerdo a la OMS una planta medicinal es definida como cualquier especie vegetal que contiene sustancias que pueden ser empleadas para propósitos terapéuticos o cuyos principios activos pueden servir de precursores para la síntesis de nuevos fármacos (Serra, 2001).
Con base en estos hechos, la OMS ha promovido el estudio de las plantas como fuente de medicamentos, dentro del programa "Salud para todos en el año 2000". Durante la conferencia de Alma-Ata, celebrada en 1978, se acordó impulsar la documentación y evaluación científica de las plantas utilizadas en la medicina tradicional, abriendo las puertas al diálogo entre la medicina tradicional y la moderna, sobre la base de que las prácticas peligrosas se eliminarían y sólo se promovería lo que fuese seguro y eficaz (Prieto, 2005).

La investigación sobre el uso de plantas medicinales forma parte de la etnobotánica, que ha sido definida como el estudio de las interrelaciones entre los grupos humanos y las plantas. Por su naturaleza interdisciplinaria abarca muchas áreas, incluyendo: botánica, química, medicina, farmacología, toxicología, nutrición, agronomía, ecología, sociología, antropología, lingüística, historia y arqueología, entre otras; lo cual permite un amplio rango de enfoques y aplicaciones (Arrillaga, 1996). No obstante, aunque existen excepciones notables, muchos investigadores incursionan en este campo de estudio desde el ámbito de sus propias disciplinas.

A pesar del interés común, ha existido poco intercambio de teorías y métodos entre disciplinas. (Álvarez, 2007). Tal situación ha favorecido una alta proporción de estudios etnobotánicos descriptivos, limitados a compilar listas de plantas útiles. Esto ha contribuido a una percepción negativa de la etnobotánica, la cual ha sido vista como una pseudociencia que carece de un contexto teórico unificado y de técnicas de análisis rigurosas (Peña, 2007).

En las dos últimas décadas se ha hecho un esfuerzo importante para cambiar esta percepción. En ese sentido, la utilización de técnicas cuantitativas ha permitido valorar con mayor precisión la importancia relativa de las plantas en contextos culturales concretos y los patrones de variación 
del conocimiento tradicional dentro de las comunidades locales; los estudios en diferentes grupos étnicos latinoamericanos han documentado experiencias de manejo que podrían constituir la base para diseñar estrategias de conservación y manejo sostenible de ecosistemas tropicales. (Eymann, 2009). Igualmente, algunas técnicas ecológicas han resultado útiles para evaluar el impacto ecológico de la extracción de plantas útiles en comunidades naturales. Asimismo, se ha comenzado a prestar atención al problema de la propiedad intelectual del conocimiento tradicional y al desarrollo de estrategias para retribuir a las comunidades locales por su participación en las investigaciones etnobotánicas (Herrera-Mendoza y Morales, 2013).

A pesar de todas estas innovaciones, plantea que la filosofía de la etnobotánica no ha cambiado mucho, pues en la mayoría de las investigaciones sobre plantas medicinales se sigue enfatizando la documentación científica de las plantas y sus usos para beneficio casi exclusivo de grandes transnacionales, con poco interés en la dinámica de los sistemas de conocimiento local y en la compensación a las comunidades nativas (Ruíz, 2005). Se requiere entonces de más trabajo interdisciplinario, de una mayor preocupación por los aspectos éticos de la comercialización de medicamentos desarrollados a partir del conocimiento tradicional de ciertos grupos humanos y por el retorno de los resultados obtenidos, en ensayos biológicos de plantas tropicales, a los países y grupos humanos que han colaborado en la colección de las plantas evaluadas. (Alfonso E, 2002).

Con base en estas consideraciones, en la presente revisión se analizan los objetivos y enfoques actuales de la investigación etnobotánica, así como las fases que debería cumplir cualquier proyecto interdisciplinario de investigación sobre plantas medicinales (Díaz, 2013).

\section{Competencias lectoescritoras}

Se considera que las competencias lectoescritoras permite según Sánchez (2014) "ejercer con eficacia los procesos de lectura y escritura o, de forma más sencilla, la capacidad para identificar, entender, interpretar y construir enunciados o textos". Si bien es cierto son la lectura y la escritura quienes tienen sus características específicas, y que se llevan a la practica en la medida en la que se da el proceso de aprendizaje. Es la lectura y a escritura un proceso que va de la mano, no aislado, cuyo propósito es el desarrollo de habilidades que acompañan a los individuos durante toda su vida.

\section{Metodología}

El proyecto se llevará a cabo desde la investigación cualitativa, pues tal como lo expresa Hernández (2014) es a través de esta que se puede comprender e interpretar la realidad del objeto estudiado. El alcance es descriptivo, bajo el modelo acción participación en donde la participación tanto de investigadores como participantes debe ser completamente activa.

\section{Técnica e instrumentos de recolección de la información}

Las técnicas para la recolección de la información fueron la entrevista semiestructurada, la observación participante, empleando el diario de campo y guion de entrevista.

\section{Escenario y actores.}

Durante el estudio participaron 200 estudiantes de la Institución Educativa Técnica Departamental San José, distribuidos en los cursos pertenecientes a la básica primaria y básica secundaria de la Institución, con edades que oscilaron entre los 4 y 17 años. 


\section{Procedimiento}

Momento (1): Diagnóstico inicial por parte de los investigadores.

Momento (2): Escogencia de las categorías que soportaran el estudio

Momento (3): Inicio de la trayectoria de indagación sobre la creación de huertas medicinales.

Momento (4): Implementación de las actividades de campo.
Momento (5): Resultados iniciales.

Momento (6): Articulación con las necesidades académicas de la IED.

Momento (7): Conclusiones.

\section{Resultados}

A continuación se presentan las actividades que permitieron la creación de la huerta medicinal:

\begin{tabular}{|c|c|c|c|}
\hline ACTIVIDADES & ESTRATEGIA & PARTICIPANTES & LOGRO ALCANZADO \\
\hline Diagnostico & Salida de Campo & $\begin{array}{l}\text { Estudiantes y } \\
\text { Docentes }\end{array}$ & $\begin{array}{l}\text { Los estudiantes distinguen las } \\
\text { problemáticas relevantes de estudiar e } \\
\text { investigar en sus alrededores. }\end{array}$ \\
\hline $\begin{array}{l}\text { Socialización del } \\
\text { diagnostico }\end{array}$ & Debate abierto & $\begin{array}{l}\text { Estudiantes y } \\
\text { Docentes }\end{array}$ & $\begin{array}{l}\text { Los maestros y estudiantes } \\
\text { participantes sensibilizan a la } \\
\text { comunidad estudiantes de la } \\
\text { necesidad de crear una huerta } \\
\text { medicinal. }\end{array}$ \\
\hline $\begin{array}{l}\text { Etapa de iniciación } \\
\text { de la huerta escolar }\end{array}$ & Espacio abierto & $\begin{array}{l}\text { Estudiantes y } \\
\text { Docentes }\end{array}$ & $\begin{array}{l}\text { Los participantes ponen en práctica la } \\
\text { fase inicial de creación de la Huerta, } \\
\text { en lo relacionado con la preparación } \\
\text { del terreno, escogencia de las plantas } \\
\text { medicinales etc. Además de socializar } \\
\text { ubicación, el proceso para la obtención } \\
\text { de recursos, los criterios de evaluación } \\
\text { del proyecto, los valores éticos a } \\
\text { fortalecer en los participantes y en } \\
\text { general las expectativas y alcances del } \\
\text { proyecto. }\end{array}$ \\
\hline Etapa de desarrollo & Trabajo de campo & $\begin{array}{l}\text { Estudiantes y } \\
\text { Docentes }\end{array}$ & $\begin{array}{l}\text { Finalizadas las tres etapas iniciales } \\
\text { se puso en marcha el proyecto en } \\
\text { el sitio escogido para el mismo, se } \\
\text { adecuó y se procedió a recolectar y } \\
\text { sembrar las plantas, se clasificaron y } \\
\text { se inició la elaboración del manual de } \\
\text { propiedades, procedimientos, usos y } \\
\text { recomendaciones o advertencias. }\end{array}$ \\
\hline Etapa de evaluación & Taller & $\begin{array}{l}\text { Estudiantes y } \\
\text { Docentes }\end{array}$ & $\begin{array}{l}\text { Dadas sus características el proceso } \\
\text { de evaluación del proyecto fue } \\
\text { permanente en cada una de sus etapas } \\
\text { a través de un "monitoreo" que se hizo } \\
\text { durante el desarrollo de cada una de } \\
\text { éstas con el fin de detectar a tiempo } \\
\text { las debilidades y obstáculos que se } \\
\text { presentaban a medida que avanzaba el } \\
\text { proyecto; cada etapa fue objeto de un } \\
\text { seguimiento en cuanto a las acciones, } \\
\text { recursos y tiempos de desarrollo. }\end{array}$ \\
\hline
\end{tabular}




\begin{tabular}{|c|c|}
\hline DIMENSIONES & RESULTADO \\
\hline Cognoscitivas & $\begin{array}{l}\text { Además del estudio de conceptos específicos referentes a las Ciencias Naturales y } \\
\text { el manejo de vocabulario botánico básico como: Métodos de reproducción, siembra, } \\
\text { trasplante, poda, abonado, entre otros, que son muy importantes para el correcto } \\
\text { desarrollo de las actividades, los participantes del proyecto fortalecerán conceptos } \\
\text { matemáticos referentes a medición de magnitudes (altura, diámetro, profundidad, } \\
\text { gramos, miligramos, litros, etc.); informáticos y lingüísticos, en la elaboración del } \\
\text { manual de propiedades y uso de las plantas. }\end{array}$ \\
\hline Psicosociales & $\begin{array}{l}\text { El proyecto de La Huerta Medicinal, tal como está diseñado, permitió abrir espacios } \\
\text { para la participación y sana convivencia a través del trabajo en equipo, desarrollar } \\
\text { y fortalecer valores éticos, el establecimiento de compromisos personales y grupales, } \\
\text { la autoevaluación y coevaluación, el desarrollo del sentido de pertenencia hacia } \\
\text { nuestras tradiciones culturales y la protección del entorno natural y en general } \\
\text { para el fortalecimiento de la formación integral de sus participantes. }\end{array}$ \\
\hline
\end{tabular}

\section{Discusión}

Si bien es cierto potenciar las competencias lectoescritoras en los estudiantes debe ser una de los objetivos fundamentales de los docentes, especialmente en el área de español, y de las estrategias que se implementen dependerá el éxito de las mismas, por lo tanto, con la creación de la huerta medicinal se tuvo en cuenta inicialmente que es lo que les interesa a los estudiantes, de acuerdo a su cultura, contexto y rol en el medio.

Conocer las especificaciones de crear una huerta medicinal, revisar la literatura frente a la temática, permitió en los estudiantes fortalecer competencias lectoescritoras, asumir roles dependiendo de las fortalezas, socializar las experiencias vivenciadas sin lugar a duda contribuyeron al logro del objetivo inicial.

Por otro lado, son las huertas medicinales las que se convierten en una alternativa para las medicinas sintéticas que generan efectos adversos sobre el organismo, sin llegar a reemplazarlas. Por esto es necesario que para algunos casos se recurra a esta, pues contribuyen al bienestar de la persona al constituir una medicina de origen natural. Con la huerta, se logró además que los estudiantes aprendieran sobre el cultivo y cuidado de las plantas.

Se logró realizar el montaje del huerto medicinal en la Institución Educativa Técnica Departamental San José del municipio de Sitio Nuevo, Magdalena; además se educó a los estudiantes para que entendieran la importancia y función de las plantas medicinales, las cuales buscan ser una alternativa a la medicina sintética que muchas veces trae consigo efectos secundarios, sin reemplazarla. Algunas de las plantas aún se encuentran en proceso de crecimiento y requieren de un cuidado, el cual es dado por los estudiantes supervisados por los docentes. Se potenciaron habilidades de escritura y literaturas asociadas directamente con la huerta medicinal, no obstante, se deben seguir realizando actividades interdisciplinarias que trasciendan del aula de clases al medio de interacción con el que habitualmente frecuentan los estudiantes.

\section{Referencias}

Acharán, M. L. (1999). La medicina natural al alcance de todos. Buenos Aires: Kier.

Alfonso, E. (2002). Curso de medicina natural en cuarenta lecciones. Buenos Aires: Kier.

Álvarez, C. y Stella, L. (2007). El uso de medicina alternativa y medicina tradicional en Medellín, 2005. Revista Facultad Nacional de Salud Pública, 25(2), 100-109.

Arrillaga de Maffei, B. (1996). Plantas usadas en medicina natural. Montevideo: Agropecuaria Hemisferio Sur SRL.

Ávila, J., Jiménez, G., González, B., Morón, F. y Fernández, R. (2008). Reacciones adversas a medicamentos herbarios y otras formas de medicina natural y tradicional en Cuba durante 2001-2004. Revista Cubana de Plantas Medicinales, 13(1). 
Cortés-Peña, O. (2016). Sustainable development in synergistic relationship with proambient behavior and fair trade. IJMSOR, 1(1), 54-58. Retrieved from http://ijmsoridi. com/index.php/ijmsor/article/view/78

Díaz, R. (2013). Algo más sobre medicina natural y tradicional. Revista Cubana de Salud Pública, 39(4), 808-813.

Eymann, A., Bellomo, M. M., Catsicaris, C. y Wahren, C. (2009). Utilización de medicina alternativa o complementaria en una población pediátrica de un hospital de comunidad. Archivos argentinos de pediatría, 107(4), 321-328.

García, J. (2013). Consideraciones sobre la Medicina Natural y Tradicional, el método científico y el sistema de salud cubano. Revista Cubana de Salud Pública, 39(3), 540555.

Guillaume, V., Marín, M., Morales, E., \& Matos, N. (2012). Conocimiento y aplicación de la medicina natural y tradicional por profesionales y técnicos de la salud. Revista Cubana de Estomatología, 49(2), 89-98.

Hernández, D. y Escobar, A. (2017). Modelo de contabilidad social como herramienta de gestión para la responsabilidad social empresarial. IJMSOR, 2(1), 44-56. Recuperado de http://ijmsoridi.com/index.php/ijmsor/ article/view/86

Herrera-Mendoza, K. y Morales, E. (2013). Perspectiva de la ecología en la comprensión de los comportamientos ambientales. Revista Omnia, 19(3). 20-30.

Largo, R. J. (1997). Medicina natural: Los diagnósticos alternativos. Madrid: Libsa.

Largo, R. J. (1997). Medicina natural: La curación por el agua, hidroterapia y crenoterapia. Madrid: Libsa.

Lezaeta, M. (1997). Medicina Natural al alcance de todos. Oaxaca: $\mathrm{X}$ pert press.

Nieves, J. y Salazar, F. (2016). e-Supply chain management para una empresa de tecnología. IJMSOR, 1(1), 31-34. Recuperado de http://ijmsoridi.com/index.php/ijmsor/article/view/74
Nogales-Gaete, J. (2004). Medicina alternativa y complementaria. Revista chilena de neuro-psiquiatría, 42(4), 243-250.

Peña, A. y Paco, O. (2007, March). Medicina alternativa: intento de análisis. Anales de la Facultad de Medicina, 68(1). 87-96.

Peña, R. y Torres, E. (2002). Algunas consideraciones sobre el empleo de productos naturales en la medicina natural y tradicional. Monografía. Bayamo, 2-6.

Prieto, A., Auró, A., Fernández, A. y Pérez, M. (2005). El empleo de medicina natural en el control de enfermedades de organismos acuáticos y potencialidades de uso en Cuba y México. Tip Revista Especializada en Ciencias Químico-Biológicas, $8(1)$

Rojas, F. (2013). En defensa de una medicina natural y tradicional avalada por la ciencia. Rev Cubana Salud Pública, 39(4). 623- 626 .

Rojas, F., Silva, L., Sansó, F. y Alonso Galbán, P. (2013). El debate sobre la Medicina Natural y Tradicional y sus implicaciones para la salud pública. Revista cubana de salud pública, 39(1), 107-123.

Ruiz, A. (2005). Aloe Vera:¿ Respalda la evidencia científica las cualidades que le atribuye la medicina natural? Metas de enfermería, 8(1), 21-22.

Sagastume, A. y Cabello, J. (2017). La educación superior y una producción más limpia. IJMSOR, 2(1), 4-8. Recuperado de http://ijmsoridi.com/index.php/ijmsor/article/view/79

Sepúlveda, A., Martínez, R., Medina, S. y Salazar, F. (2016). Propuesta de diseño de una red supply chain para la agrocadena de cacao, municipio de Viotá, Cundinamarca. IJMSOR, 1(1), 35-42. Recuperado de http://ijmsoridi.com/index.php/ijmsor/ article/view/75

Serra, B. y Lluís, J. (2001). Gran enciclopedia de las plantas medicinales: medicina natural del tercer milenio/Josep Lluís Berdonces I Serra. Madrid: Tikal. 